\title{
CAMBIOS AL CURRÍCULUM DE LA FORMACIÓN INICIAL DE PROFESORES: RESIGNIFICANDO EL SABER PEDAGÓGICO
}

\section{Paula Guerra Z.*}

RESUMEN

En esta reflexión, se proponen algunas directrices curriculares, en el contexto de la formación inicial de profesores en que el saber pedagógico es la base de su rol profesional. A partir de diversas conceptualizaciones, se propone que su construcción se debiera abordar desde una vinculación entre teoría y práctica, el desarrollo de procesos de reflexión entre futuros docentes y en una vinculación entre pedagogía y disciplina que se funda en el conocimiento pedagógico del contenido. Se discute su integración curricular y los desafíos que implica.

Palabras clave: formación inicial de profesores, saber pedagógico, reflexión docente, vinculación teoría-práctica, conocimiento pedagógico del contenido, profesionalización docente.

\section{CHANGES TO THE CURRICULUM OF INITIAL TRAINING OF TEACHERS: RESIGNIFYING TEACHING KNOWLEDGE}

\begin{abstract}
This reflection proposes some curricular ideas in the context of the teachers education, in which pedagogical knowledge is the base of the professional role of teachers. From diverse conceptualizations of this one, is propose that the construction must be approached from a links between theory and practice, the process development of reflection between students and a link between pedagogy and discipline that is founded on the pedagogic knowledge of the content. This curricular integration is discussed and the challenges that implies.
\end{abstract}

Keywords: teacher education, pedagogical knowledge, teacher reflection, link practice-theory, pedagogic knowledge of the content, teacher professionalization

* Magíster en Psicología educacional. Programa de Doctorado en Ciencias de la Educación. Pontificia Universidad Católica de Chile.pfguerra@uc.cl 


\section{Introducción}

Los continuos cuestionamientos a la calidad de la educación nos enfrentan día a día a la necesidad de reflexionar y analizar las causas de estos problemas.

La calidad de la educación depende de múltiples factores. Pero, sin duda, un actor fundamental es el profesor $y$, por tanto, para avanzar hacia una educación de mayor calidad, es primordial revisar la calidad de los procesos de formación de los docentes; en este caso, enfocando la mirada hacia su formación inicial.

El presente trabajo, tiene por objetivo reflexionar críticamente con respecto a las deficiencias de los currículos de formación inicial, asumiendo la complejidad de esta sociedad y las demandas asociadas a la educación, enfocándose específicamente en el saber pedagógico, elemento fundamental para una docencia acorde a la nueva sociedad. Si bien se reconoce que éste es un aspecto central, los esfuerzos en esta línea no han sido suficientes, por lo que es necesario asumir una mirada en que este saber sea el eje articulador del currículum de la formación de profesores. Algunos de los ámbitos sobre los cuales se podría enfatizar se organizan en torno a 3 ejes centrales: la vinculación teoría y práctica, la reflexión entre futuros profesores y el conocimiento pedagógico del contenido.

A continuación, se presentarán los antecedentes y formulación del problema, la tesis que se propone para abordarla, para finalizar con las principales conclusiones y discusiones respecto al tema.

\section{Antecedentes del problema}

Los últimos tiempos han experimentado avances complejos y rápidos. Nuestra sociedad está sometida a procesos de transformación que están afectando la forma en que nos organizamos, trabajamos y aprendemos. Estos cambios tienen un impacto directo en la escuela, en tanto institución encargada de formar a los nuevos ciudadanos (Marcelo, 2002). 
Una de las consecuencias de este cambio social y cultural, es que la información con que día a día cuentan los alumnos y profesores aumenta vertiginosamente, al igual que su acceso (Murillo, 2002), lo cual supone nuevos desafíos a la educación.

Además, si consideramos el avance sustantivo que han tenido las llamadas tecnologías de la información y comunicación, es altamente probable que gran parte de la adquisición de la información transcurra fuera de sistemas institucionales, como la escuela. Si no se cuestiona esta realidad, el sistema educativo tenderá a perder relevancia y valor, y las personas buscarán habilidades y conocimientos fuera del sistema formal, probablemente de modo desordenado y aleatorio (Ottone \& Hopenhayn, 2007).

Esta nueva sociedad, y los desafíos que conlleva, impactan de manera significativa en los docentes, quienes deben asumir la complejidad del conocimiento, los nuevos medios para adquirir la información, las habilidades necesarias para trabajar con ella, etc. Sin embargo, la presente formación de los profesores parece no actualizarse con la misma rapidez de estos cambios y no logra responder a este tipo de desafíos.

Al análisis de los desafíos que el cambio social y cultural supone para las escuelas, se deben sumar los problemas ampliamente difundidos de la calidad de la educación, evidenciado en los bajos resultados de aprendizaje de los alumnos en diversas pruebas nacionales e internacionales (Eyzaguirre \& Le Foulon, 200I; Informe Nacional SIMCE, 2007). Estos resultados muestran que los alumnos no aprenden comprensivamente, no demuestran un desempeño flexible que les permita realizar múltiples operaciones con el conocimiento (explicarlo con sus propias palabras, compararlo con otros contenidos, conectarlo con otros aprendizajes, etc.). Los resultados de las pruebas SIMCE tomadas en los últimos años muestran un estancamiento, algunas mejorías en algunos casos y leves retrocesos en otros (MINEDUC, 2006). La prueba PISA 2000, muestra que los estudiantes chilenos tienen un rendimiento muy inferior a aquellos con quienes deberán competir en el futuro. 
Frente a estas realidades, los cuestionamientos a la calidad de los profesores son recurrentes. Sin embargo los profesores que actualmente se encuentran en servicio, ¿han sido formados para hacer frente a los enormes desafíos que implica esta nueva sociedad?

La respuesta parece ser negativa. Es necesaria una nueva visión del profesor acorde a los nuevos tiempos. Una visión del profesor como profesional de la enseñanza, tal como se puede visualizar en la siguiente cita:

El profesionalismo docente no es sólo una etiqueta formal. Es una exigencia de la sociedad del conocimiento, de la complejidad creciente de la educación escolar y del carácter estratégico que ésta ha adquirido. La proyección del quehacer docente no se limita a la escuela ya que su misión se enclava, también, en distintas instancias societales que requieren ser atendidas pedagógicamente, como son las necesidades emergentes de niños, jóvenes y adultos, derivadas de múltiples factores que caracterizan nuestra época. (Comisión sobre formación inicial docente, 2005, p. 17).

Esta afirmación ilustra la complejidad creciente de la tarea docente, que supone, al menos, una revisión a la formación de los futuros profesores. ¿Están las instituciones formadoras de profesores asumiendo una visión profesionalizante de la tarea docente?

\section{Características de la Formación Inicial}

A partir de los inicios de la década del 70, luego del cierre de las Escuelas Normales, la formación de profesores en Chile pasa a ser enteramente de carácter terciario, a cargo de Universidades e Institutos Profesionales. A raíz de la dictación de la Ley Orgánica Constitucional de Enseñanza (LOCE, I989), la formación de profesores debe conducir a la obtención del grado académico de licenciado y al título profesional, pudiendo ser impartida solamente por universidades y por aquellos institutos profesionales que tuviesen carreras de pedagogía creadas con anterioridad a la LOCE. 
En Chile, existen tres modalidades de formación de docentes. Primero, la modalidad de formación concurrente, que es la más frecuente en los programas de formación de profesores para la enseñanza Parvularia, Básica y Media. Consiste en el desarrollo de un currículo que simultáneamente ofrece una formación general, una formación específica o de especialidad acorde al nivel escolar en que se desempeñarán sus egresados y una formación pedagógica. Si bien la proporción de créditos u horas destinadas a cada uno de estos ámbitos difiere mucho de una institución a otra, en todos los casos se imparten asignaturas de los tres ámbitos. Las carreras de este tipo tienen, generalmente, una duración de ocho a diez semestres y exigen jornada intensiva, excepto en un número reducido de carreras que se imparten en jornada vespertina. Segundo, la formación consecutiva, orientada a personas que tienen una licenciatura académica o un título profesional centrado en alguna disciplina específica y que desean obtener un título de Profesor de Enseñanza Media. Para ello, se imparten carreras de dos a cuatro semestres de duración, centradas en temas pedagógicos y didácticos, propios de las distintas especialidades. En esta misma categoría, se inscriben los programas de formación de docentes para ejercer en la enseñanza media técnicoprofesional.

Tercero, la modalidad de programas especiales, entre los cuales se distinguen: I) los programas de regularización, destinados a personas que tienen estudios incompletos de pedagogía o bien una práctica docente prolongada, los que ofrecen la posibilidad de lograr un título profesional en un tiempo menor al habitual; 2) los programas abiertos a personas que solamente han cursado Enseñanza Media y que, en algunos casos, tienen alguna experiencia de trabajo o colaboración en establecimientos educacionales en tareas no docentes.

La formación de profesores en Chile se relaciona con los niveles o modalidades del sistema escolar y toma en consideración los sectores de aprendizaje del currículo escolar, dando origen a distintas carreras pedagógicas para formar docentes de educación Parvularia, Básica, Diferencial y Media. No obstante, en virtud de la autonomía 
universitaria, las instituciones de educación superior son libres de diseñar sus propias carreras. Así, por ejemplo, algunas universidades ofrecen carreras que apuntan al ejercicio profesional tanto en Educación Parvularia como en los primeros años de Educación Básica; otras instituciones están ofreciendo formación de profesores de algunos subsectores, como Inglés o Educación Física, habilitados para desempeñarse tanto en educación básica como media; y, por otra parte, las universidades han formado tradicionalmente de maneras distintas a los docentes de humanidades y del área científica; en este último caso, pueden hacerlo diferenciadamente según las especialidades de: matemática, biología, física y química, o bien, agrupando las tres últimas bajo la denominación amplia de ciencias naturales, o agrupando dos de las tres disciplinas mencionadas.

\section{Nudos críticos en la formación docente}

Cuando se cuestiona la calidad de los docentes, es recurrente también cuestionar la calidad de los programas de formación de éstos. Al respecto, existen diversas evaluaciones que han mostrado nudos críticos que requieren ser urgentemente abordados.

Por ejemplo, el informe de la OECD (2003) cuestiona aspectos clave de la formación, especialmente la difícil y compleja articulación que ha habido en el currículum, entre el saber pedagógico y el saber disciplinar, la distancia con la realidad escolar y la compleja relación entre teoría y práctica, entre otros aspectos. Al respecto menciona:

En general, los profesores de enseñanza básica en Chile terminan enseñando el conocimiento sobre matemáticas y lenguaje que adquirieron en la enseñanza media. Puesto que los estudiantes de $8^{\circ}$ básico, tienen tan bajos puntajes en matemáticas y comprensión de lectura en los exámenes internacionales, esto sugiere que los profesores chilenos ingresan a la docencia con niveles de conocimiento de la materia bastante bajos en comparación con los profesores de países desarrollados y de muchos países en desarrollo. (OECD; 2003, p. I40) 
Además, considera que la formación inicial de profesores de educación básica actualmente no proporciona suficientes cursos de contenidos en matemáticas, lenguaje y otras materias en la malla curricular de formación inicial, incluso para profesores de cursos del primer ciclo, pero la falta de estos cursos constituye un agudo problema cuando los profesores de enseñanza básica terminan enseñando en el segundo ciclo básico.

Terminan planteando, que las instituciones formadoras de profesores necesitan enseñar más cursos de contenidos disciplinares a los profesores de educación básica y vincular estos cursos con el currículum de la reforma.

Desde esta visión, se recoge una dificultad derivada de la débil articulación entre el conocimiento disciplinar y la formación de profesores. Sin embargo, ésta parece ser una mirada un tanto restringida frente a las necesidades curriculares en este ámbito. Torres (I996) menciona el énfasis que tradicionalmente ha existido en la formación científica o disciplinar, como una de las características de la formación inicial latinoamericana y que el debate respecto a su necesidad es permanente. Nervi (2007) sostiene que este fenómeno es una revitalización del antiguo conflicto entre el saber disciplinario especializado y el saber pedagógico. Además, este es un tipo de formación que responde a una lógica academicista (Moreno, et al., 2006).

Por otra parte, el documento elaborado por la comisión para la formación inicial docente (2005) recoge otro tipo de preocupaciones. Dentro de los principales nudos críticos, menciona que la conceptualización del saber pedagógico no es un eje articulador de la formación docente inicial. Se trabaja en espacios fragmentados, con mallas de formación en que predomina un itinerario formativo sobre la base de la acumulación de actividades curriculares, sin vinculación entre sí.

Estos dos documentos (OECD, 2003; Comisión para la formación inicial docente, 2005) enfocan el problema de la formación inicial 
docente, desde dos lógicas distintas, planteando a su vez, una tensión frecuentemente debatida en este ámbito: ¿debe predominar la formación disciplinar de un contenido o la formación eminentemente pedagógica? ¿Se pueden conjugar o articular de alguna forma estos dos aspectos de la formación, o debe predominar uno sobre otro?

Una investigación con respecto al saber pedagógico (Valenzuela, 2006) revela problemas en lo relativo a este tipo de saberes. Sitúa el origen de esta situación en una formación inicial deficitaria, que no facilita la construcción del saber pedagógico como base y sustento del ejercicio profesional. Dicha transformación, sólo será posible en la medida que los programas de formación inicial reduzcan su orientación técnica instrumental y den cabida a procesos de reflexión participativa, donde los futuros profesores efectivamente sean protagonistas y construyan su saber especializado, a través del ejercicio colegiado y autónomo.

Carranza y Reyes ${ }^{\mathrm{I}}$ (2005), mencionan que la incorporación del saber pedagógico en los programas de formación se ha visto dificultada por una estructura curricular fragmentada, marcada por divisiones disciplinarias que no favorecen una visión integrada del saber. Además, se refieren a la escisión entre el mundo académico y el escolar, que se ha traducido en la exclusión de los maestros, de la posibilidad de legitimar y construir un saber profesional basado en la propia experiencia, distanciando a la práctica de su rol como fuente constitutiva de las ciencias de la educación. Incluso mencionan que, por estas razones, el saber pedagógico no está presente en los programas de estudio de las carreras pedagógicas.

\section{El saber pedagógico}

La conceptualización del saber pedagógico no es un tema sencillo. Son múltiples sus interpretaciones e, incluso, las propias instituciones formadoras no tienen una visión unificada al respecto (Carranza \&

I En un documento que sistematiza la discusión que se generó en el segundo encuentro nacional sobre formación docente "Saber Pedagógico y propuestas de políticas". 
Reyes, 2005; González², 2006). En los documentos que sistematizan la visión del saber pedagógico de las instituciones formadoras de profesores, se sostiene que, en general, en las instituciones formadoras de profesores, predominan tres lógicas: una técnica e instrumental, en donde existen conocimientos formalizados y estructurados en la cultura académica; otra, donde el conocimiento se asume como dialógico e intersubjetivo, alejándose de la perspectiva técnica, para situarse en un proceso de transacción cultural; y una última que entiende el conocimiento como eje articulador de las relaciones entre prácticas y saberes pedagógicos. Estas diferentes miradas tensionan la conceptualización de este saber, ya que se aprecia una dificultad de armonizar una racionalidad instrumental y otra de orden valórico y con orientaciones que obedecen a supuestos epistemológicos diferentes: saber o conocimiento; naturaleza teórica o práctica; transmisión de conocimiento formalizado o construcción reflexiva del mismo.

Existen visiones extremadamente simplificadoras del saber pedagógico, como la del Banco Mundial (1995), que lo reduce a un repertorio de habilidades de enseñanza o a la acción de planificar y evaluar. Sin embargo, desde otras visiones, el saber pedagógico es mucho más complejo.

Díaz (2005) plantea que el saber pedagógico está constituido por conocimientos construidos de manera formal e informal por los docentes; valores, ideologías, actitudes, prácticas; es decir, creaciones del docente, producto de las interacciones personales e institucionales, que evolucionan, se reestructuran, se reconocen y permanecen en la vida del docente. El saber pedagógico es teoría y práctica; la primera se logra a través de las reflexiones conscientes o inconscientes de los docentes y esa producción teórica fundamenta la práctica, que a su vez prueba la teoría.

Para Ibáñez (2007), el saber pedagógico es siempre situado y dinámico. Se nutre de otras disciplinas y se reconstruye en la práctica

2 Informe redactado por Loreto González, docente de la Universidad Academia de Humanismo Cristiano y en representación de la RIPU (Red de innovación pedagógica universitaria) 
pedagógica. El núcleo de este saber es el aprendizaje del estudiante y se nutre también de un conocimiento más general, sobre su contexto, la escuela y el curriculum, además de un saber sobre el contexto interaccional apropiado en el aula. Incluye también el conocimiento y la comprensión del contenido y de la didáctica de cada especialidad. En definitiva, considera que este saber es una conjunción de aspectos que no pueden darse desarticulados.

Una propuesta más amplia es la que realizan Moreno et al. (2006), quienes consideran cuatro dimensiones constitutivas de este saber: disciplinar, procedimental, estratégico y ético-político. El primero de estos niveles se refiere a los contenidos del área, su selección, conocimiento disciplinar y didáctico. El segundo, al diseño y planificación de la actividad educativa, la selección de materiales, evaluación, rutinas, etc., y el tercero, al conocimiento de los sujetos y su contexto, la resolución de conflictos y el manejo de situaciones contingentes. El último, por su parte, se adscribe a un sentido referente a la labor educativa, los valores que hay detrás y la intersubjetividad de esta tarea. Dentro de esta lógica, el saber pedagógico se configura en la interrelación mutua entre estas cuatro dimensiones.

La importancia del saber pedagógico en este momento histórico es básica. Si consideramos que el contexto social y cultural demanda nuevos roles a la educación y específicamente a los docentes, que el conocimiento día a día crece, que es necesario contar con habilidades de orden superior para utilizar cantidades crecientes de información, etc., la exigencia por una verdadera profesión docente es una de las grandes demandas de la sociedad. Se necesitan profesores que funden su saber sobre un conocimiento propio, científicamente construido y con una sólida articulación con la práctica y que sea terreno exclusivo de los profesionales de la educación.

Las instituciones formadoras revelan diversas propuestas curriculares, coherentes con las diversas conceptualizaciones que realizan del saber pedagógico. Sin embargo, resalta explícitamente la línea de práctica, como espacio que promueve la reflexión y vinculación teoría-práctica. Pese a esto, llama la atención que, desde el carácter transversal que 
le atribuyen algunas instituciones al saber pedagógico, aparecen experiencias que tenderían a reforzar una cierta invisibilidad del mismo. Se menciona que, "en la actualidad, hay indicios de que el saber pedagógico está incorporado en la formación pedagógica y de la especialidad básicamente como saber "consumido", puesto que, lejos de promover la generación de conocimiento en los procesos formativos, la tendencia parece ser, en los hechos, reducirla" (González, 2006, p. 7).

Una completa revisión de diversas experiencias de práctica en formación de profesores de diversas especialidades o menciones es presentada por Moreno et al., (2006). Al respecto, estos investigadores sostienen que la reflexión sobre los discursos y propósitos de la enseñanza, sobre la disciplina y las implicancias políticas de los procesos educativos, es difusa y precaria. Es necesario generar profundos cambios en la formación y ejercicio profesional del maestro, de modo que conduzcan a la reconquista de su saber. En general, su evaluación refleja que los practicantes no construyen saber pedagógico, sino que realizan una apropiación de los saberes que circulan en la escuela.

De Tezanos (2005) manifiesta que el saber pedagógico es el fundamento del oficio de enseñar. Es ese tipo de saber que produce el maestro y por cuya producción se transforma en miembro de un colectivo. Por tanto, para esta investigadora, este saber debiera ser el eje fundacional de la formación de un maestro. Y menciona:

En tanto es el saber pedagógico el que le da identidad a la profesión docente, el que diferencia a los maestros, los transforma en intelectuales autónomos, capaces de generar sus propias búsquedas, de entregar autoridad al otro y de legitimar y validar aquellos elementos de las conceptualizaciones producidas en las formaciones disciplinarias que amplían y permiten la transformación de su práctica. Y es sólo en este espacio de la producción de saber pedagógico, donde las contribuciones de diferentes aparatos disciplinarios cobran relevancia. Puesto que es allí, en la relación entre práctica y reflexión, eje articulador del saber, donde los conceptos disciplinarios pertinentes 
son resignificados. Y es en esta instancia, en este momento, donde comenzamos a recuperar la identidad, el significado y el sentido del oficio de enseñar, es decir, del proceso de profesionalización reclamado por quienes formulan políticas educativas (p. 67).

En esta misma línea, Gauthier (2006) señala que un aspecto fundamental de la profesionalización docente reside en la identificación de los saberes de la práctica del oficio de docente. Para alcanzar esto, no basta con asimilar las competencias profesionales del docente a las competencias del especialista de una disciplina, ya que enseñar es una actividad compleja en el sentido en que implica ambigüedad, imprecisión, incertidumbre y no puede reducirse a la formación de un profesional no pedagógico.

De esta forma, la profesionalización docente, requisito fundamental para una docencia acorde a la sociedad de la información y del conocimiento, requiere un saber propio que le permita articular los diferentes ámbitos de la profesión. Este saber pedagógico no ha sido suficientemente desarrollado en los programas de formación inicial (Comisión formación inicial docente, 2005; Valenzuela, 2006), lo cual implica desafíos importantes para éstos.

En función a los argumentos anteriormente desarrollados, la pregunta que guiará esta reflexión se formula de la siguiente forma:

\section{Considerando que el saber pedagógico debiera constituirse en el eje articulador del currículum de la formación inicial de profesores, ¿qué elementos debiera considerar éste para abordar con éxito este desafío?}

La revisión de las diversas conceptualizaciones del saber pedagógico permite, a partir de ellas, reelaborar y construir la visión del saber pedagógico que se utilizará en esta argumentación. En primer lugar, es necesario considerar que el saber pedagógico es una construcción social y personal sobre múltiples aspectos de la práctica docente, tanto del aprendiz, su contexto, como las interacciones educativas. Es teoría y práctica y se asocia a una reflexión continua. Se funda en diversos 
saberes que se articulan en la práctica, siendo por tanto, mucho más que conocimiento pedagógico o disciplinar. En tanto, el saber específico de la profesión, otorga identidad al docente y por tanto permite, en parte, su profesionalización. Por lo tanto, y dada su centralidad en la labor docente, los programas de formación de profesores debieran considerar elementos que faciliten la construcción de este saber.

Desde esta mirada del saber pedagógico, los programas de formación debieran situar este saber como eje articulador de su propuesta curricular, en función de una vinculación entre teoría y práctica, el desarrollo permanente de la reflexión entre futuros profesores y un conocimiento pedagógico de la disciplina.

Como primer punto, es importante plantearse que un programa de formación de este tipo debiera situarse como un modelo centrado en el análisis (dentro de la propuesta de Ferry, I99I, en Moreno et al., 2006), esto es, un tipo de programa en donde el énfasis está en lo impredecible e incontrolable de la labor docente y en asumir una articulación entre teoría y práctica, en donde la primera no puede tener valor formativo si se aleja de la práctica; y la segunda no puede ser formadora por sí misma si no es leída con un referente teórico.

Dentro de esta lógica, Gauthier (2006) menciona la necesidad de la puesta en marcha de una formación integrada y anclada en los lugares de práctica. Por integración entiende el establecimiento de vínculos entre los cursos de naturaleza más teórica y los que tienen una intención más práctica, y también de vínculos con las condiciones reales del oficio.

En esa línea, desde las instituciones formadoras de profesores, el saber pedagógico se desarrolla en los programas de formación mediante líneas de práctica, que integran saberes previos y acerca al alumno a problemas reales, estructurando conocimientos que le permitan aplicarlos en su desempeño profesional (Carranza \& Reyes, 2005).

Si ampliamos el concepto de saber pedagógico y lo miramos en relación con el aprendizaje docente, debemos asumir que éste 
es situado, socialmente distribuido y mediado por objetos o herramientas (Borko \& Putman, I996, en Ávalos, 2003). Reconocer el aprendizaje docente como un aprendizaje situado significa integrar en la mejor forma posible el saber pedagógico, los conocimientos pedagógicos de la disciplina y el saber disciplinario en torno a situaciones educativas reales antes $y$, sobre todo, durante los períodos de prácticas o experiencias de enseñanza en aula (Ávalos, 2003).

La articulación entre teoría y práctica ha sido abordada principalmente mediante dos modalidades: asignaturas de prácticas y la práctica como un componente de una asignatura teórica (Contreras et al., 2008). Esta visión asume que el dominio teórico antecede al dominio práctico. Sin embargo, una versión alternativa plantea que el proceso de formación docente requiere comenzar por la inserción en un centro educativo, asumiendo que "hay que hacer docencia, para saber sobre docencia” (Contreras et al., 2008, p. 3).

Un elemento complementario al anterior es la necesidad de generar procesos de reflexión entre docentes, que permitan la generación de este saber, a partir de la práctica. En ese sentido, podemos entender que la reflexión es el medio mediante el cual se construye, desde la relación teoría-práctica, el saber pedagógico.

Nervi (2007) menciona que la valoración de la práctica da sentido a la experiencia, producto de la reflexión sobre y en la actividad práctica. Además, el saber pedagógico se constituye a partir de los diversos grados de reflexión que elabora el docente, a partir de los discursos y prácticas en una experiencia educativa (Moreno et al., 2006).

Este elemento es reconocido como central, para los diversos programas de formación de profesores, los que sostienen que "no basta con enseñar el aspecto metodológico y contenidos de una asignatura, es esencial que los futuros profesores sean capaces de reflexionar sobre las capacidades, contenidos, destrezas y valores que deben entregar a sus alumnos. Desde esta perspectiva, la formación inicial debe orientarse a procesos de reflexión, experimentación y evaluación de la acción educativa” (Carranza \& Reyes, 2005, p. 4). 
Las diversas propuestas que se han realizado sobre formación inicial (Estándares de Desempeño para la Formación Inicial de Docentes, 200I, Informe Comisión formación inicial docente, 2005, entre otros) mencionan diversas alusiones al pensamiento reflexivo de los profesores, que se asume estrechamente ligado a la profesionalización docente (Cornejo, 2003).

Dentro de las diversas conceptualizaciones de la reflexión docente, en este artículo se asume la propuesta de Jay \& Johnson (2002), que la definen como un proceso individual y colaborativo, que implica experiencia e incertidumbre. Comprende la identificación de cuestiones y elementos clave de una temática que es significativa y cuyo proceso permite lograr nuevas claridades que faciliten la modificación de la acción. Estos autores distinguen tres tipologías de reflexión: descriptiva, comparativa y crítica.

Desde esa lógica, el tema de la reflexión docente comienza a ser un aspecto clave para la formación de los profesores, ya que se articula de muy buena manera con la visión de la enseñanza como un proceso complejo, analítico e investigador. En ese sentido, se plantea la necesidad de ser conscientes de las decisiones pedagógicas que se toman y la importancia de reflexionar y analizar críticamente las prácticas ya implementadas (Harford \& MacRuairc, 2008).

Dentro de este ámbito, es importante hacer notar la referencia a la reflexión entre profesores. Desde esta noción, se potenciaría una reflexión asociada a una práctica social y no sólo individual. Cornejo (2003) sostiene la necesidad de constituir comunidades de aprendizaje docente que faciliten el mutuo apoyo y estimulación entre docentes. Además, al retomar la noción de que el conocimiento se construye socialmente, se parte de la base de la mayor riqueza en la contribución, cuando son muchos los que aportan a la solución de un problema educativo, al análisis de la viabilidad de una versus otra estrategia o a la interpretación de marcos curriculares distintos (Ávalos, 2003).

Por último, si se ha asumido que el saber pedagógico es más que conocimiento disciplinario o pedagógico, bajo qué mirada podría 
articularse la relación entre estos dos aspectos. Una respuesta a esta interrogante la ofrece Shulman (1987; 200I), mediante su concepto de conocimiento pedagógico del contenido. Al respecto, menciona que éste es una especial amalgama entre materia y pedagogía que constituye una esfera exclusiva de los maestros, su propia forma especial de comprensión profesional.

Para Ávalos (2006), comprender pedagógicamente una disciplina es comprender en profundidad sus conceptos y temas centrales. Desde los alumnos y desde el conocimiento, ser capaz de efectuar las selecciones necesarias, y poder representar estos conceptos y temas usando un repertorio de herramientas.

El conocimiento pedagógico de los contenidos adquiere particular interés, porque identifica los bagajes distintivos de conocimientos para la enseñanza. Representa la mezcla entre materia y pedagogía, por la que se llega a una comprensión de cómo determinados temas y problemas se organizan, se representan y se adaptan a los diversos intereses y capacidades de los alumnos y se exponen para su enseñanza. El conocimiento pedagógico de la materia es la categoría que, con mayor probabilidad, permite distinguir entre la comprensión del especialista en un área del saber y la comprensión del pedagogo (Shulman, I987; 200I, p. I75).

La relación entre la forma de definir y comprender el contenido y la pedagogía, así como sus relaciones, implica que, para poder ejercer la docencia, se requiere la transformación de lo comprendido de determinado cuerpo disciplinar, lo cual descansa, entre otros, en "el conocimiento profundo, flexible y cualificado del contenido disciplinar, pero, además, en la capacidad para generar representaciones y reflexiones poderosas sobre ese conocimiento" (Shulman, I999, citado en Salazar, 2005). El estudio del conocimiento pedagógico del contenido ofrece la oportunidad de entender cómo los profesores llegan a hacer enseñables los contenidos, en términos de una cierta habilidad de convertir sus comprensiones acerca de un tema, en distintas estrategias de enseñanza que faciliten el logro de los aprendizajes en sus estudiantes. 
Esta interacción entre pedagogía y disciplina es la que permite la transformación del contenido para su enseñanza. Esto sólo ocurre cuando el docente reflexiona e interpreta críticamente la información pedagógica, disciplinar y del contexto (Shulman, I987; 200I).

Por su parte Grossman (1990, citado en Salazar, 2005) señala que existen cuatro fuentes desde las cuales es posible observar la generación y desarrollo de este tipo de conocimientos: la observación de experiencias de aula, tanto como estudiante y como docente en formación; la formación disciplinar, los cursos específicos de pedagogía y la experiencia como docente ya en el aula. Estos diversos contextos influyen en la formación de este conocimiento y permiten ahondar la mirada en procesos que van más allá del restringido espacio de la formación inicial de los profesores.

\section{Conclusiones y comentarios finales}

La formación de profesores es un tema de relevancia a nivel mundial. Diversas han sido las preocupaciones que se han plasmado en documentos que tematizan la importancia de este tema para el mejoramiento de la educación. Parece ser que un elemento central y articulador de estas reflexiones se sitúa en construir un ámbito de acción propio del ser profesor, que permita entregar la necesaria identidad a un colectivo que se ha visto sometido a frecuentes críticas y cuestionamientos.

Es en ese contexto donde el saber pedagógico toma fuerza y presencia en la discusión con respecto a la formación de los docentes. Parece ser que este tipo de saber reúne en sí mismo elementos clave de lo que es "ser profesor".

Tal como se mencionó anteriormente, no existe un claro consenso para conceptualizar este saber. Sin embargo, las diversas visiones convergen en elementos centrales, entendiéndose como un conjunto integrado de diversos elementos, entre los que se encuentran conocimientos, actitudes, valores, entre otros. En ese sentido, surge una primera diferenciación con la línea de investigación anglófona, 
que sitúa este tipo de saberes como una categoría de conocimiento, refiriéndose a éste como pedagogical knowledge. Sin duda, más allá de connotaciones propias de uno u otro idioma, es necesario profundizar en lo que estas dos tradiciones están proponiendo como un saber especializado de la docencia, ya que, si se asume que el saber es un tipo de conocimiento que surge desde la experiencia y es asistemático, se le está distinguiendo del conocimiento propiamente tal, que parte de un proceso de construcción regido por leyes científicas. En ese sentido, es relevante diferenciar entre ambos conceptos, ya que los supuestos en la base de uno y otro son diferentes e incluso contradictorios.

Por otra parte, asumir el saber pedagógico como un eje articulador de la formación de profesores implica asumir también una serie de compromisos con los elementos sobre los cuales se fundan. Si bien, en la propuesta que se presenta aquí, se asume que el currículo de la formación de profesores se construye sobre un saber pedagógico, se reconoce también que ese saber no se construye en el vacío. De ahí que sea necesario que, al proponer un cambio de este tipo, se proponga, a su vez, un currículo centrado en una relación entre teoría y práctica, donde ambas alimentan este saber pedagógico y se le visualiza como un eje integrado y articulado horizontal y verticalmente en el plan de estudios. Esto implica que el curriculum, por una parte, asume la línea de vinculación teoría y práctica progresiva a partir de nudos temáticos, que se van desarrollando en el transcurso de la formación $y$, por otra, se integra verticalmente con el resto de las asignaturas, de modo de nutrir a éstas con las experiencias prácticas que se tienen, y a su vez construir saberes a partir de ella.

Sin embargo, no basta con la articulación entre teoría y práctica para construir saber pedagógico. Es necesario, además, contar con espacios de reflexión que permitan ir considerando las diversas aristas de la labor docente. De esta forma, se esperaría que el currículo trasversalizara la reflexión, no sólo en el eje de teoría y práctica, sino que como una forma de articular los diversos elementos que integra la formación, de modo de promover programas integrados, que se alejan de la compartimentalización disciplinaria, tan presente en los 
espacios formativos de la actualidad. Para esto, la reflexión resulta de gran utilidad, aunque no podemos limitar sólo en este aspecto su valor formativo, ya que se espera que ésta sea un producto fundamental de la formación de profesores, convirtiéndose en un hábito que permita la progresiva construcción de saber pedagógico en el aula.

Dentro de este mismo ámbito, se esperaría que la reflexión dentro de los programas de formación siguiera lógicas progresivas, en la misma línea de la vinculación teoría-práctica, en tanto, su nivel y objeto, se iniciara en niveles descriptivos, avanzara hacia miradas comparativas, para finalmente llegar a niveles críticos. En ese sentido, resultaría interesante establecer paralelos entre conceptualizaciones más básicas o descriptivas del saber pedagógico y niveles más superficiales de reflexión, en especial, en los inicios de la formación, para luego ir complejizándose hacia relaciones entre un saber pedagógico con tintes éticos y políticos, asociado a reflexiones críticas. Este último elemento debe intencionarse desde los primeros años, de modo que permita a los futuros profesores ir entendiendo las implicancias de su labor, en especial, en un contexto social y cultural tan competitivo e individualista. En ese sentido, es relevante investigar y profundizar más en las relaciones que se proponen, en los alcances que puede tener en una formación menos fragmentada y en los compromisos que se asumen desde las instituciones formadoras para avanzar hacia estas nuevas relaciones.

Pero el alcance de la reflexión no llega sólo hasta acá. Las relaciones entre saber pedagógico y conocimiento pedagógico del contenido se fundan en una reflexión constante de las transformaciones que se hacen de uno a otro. Es imposible apostar por una formación integradora si no se asumen las profundas relaciones que existen entre el saber pedagógico y el saber o conocimiento disciplinar. Más que recordar viejas rencillas por la supremacía de uno u otro, considero beneficioso apropiarse del concepto propuesto por Shulman, como una forma de establecer estas relaciones dentro del currículo, en el marco de una reflexión instalada en las diversas asignaturas, que permita visualizar los fundamentos epistemológicos de cada 
disciplina y su articulación con la pedagogía, y que se vea enriquecida por las implementaciones que día a día realizan los docentes, de modo de nutrirse y contrastar las aproximaciones disciplinares que se realizan en la sala de clases.

De esta forma, una propuesta que intente abordar el saber pedagógico desde estas tres aristas permitiría hacer frente a las enormes demandas que se asocian a la tarea educativa. Sin embargo, su implementación supone desafíos importantes para las instituciones formadoras, las cuales deben ser investigadas seria y acuciosamente. Por ejemplo, el rol que cabe a los formadores de profesores, en tanto modelos, hasta qué punto compartirán la visión de saber pedagógico que se propone, o más bien trasmitirían versiones simplistas de éste, que conflictuarían a los estudiantes. Por otra parte, hasta qué punto una visión de saber pedagógico puede ser impuesta a los formadores, los cuales han sido socializados en otras lógicas.

Por otra parte, en general, en este documento se ha hecho referencia al saber pedagógico en tanto elemento central de la formación de profesores, pero su relevancia va más allá incluso. Basta con mencionar el impacto que tiene, si se asume como un eje articulador de la investigación en el campo educacional. Algunas propuestas en este ámbito parten de la base de comprender la naturaleza de este saber: qué es y cuál es su objeto de estudio, cómo y quién lo produce, cuál es su estatus epistemológico. Asimismo, sería interesante investigar cómo se sitúa el saber pedagógico en el diálogo entre la educación y otras disciplinas que la estudian: es también en educación un código propio y exclusivo, excluyente de otros campos de conocimiento o, por el contrario, puede ser estudiado y enriquecido desde allí. Estas y otras preocupaciones se podrían levantar como objetos de estudio en este ámbito, surgiendo como insumos importantes en la clarificación de un concepto tan valioso, pero a su vez escasamente consensuado.

Una última reflexión, o más bien cuestionamiento, que surge a partir de la revisión y propuesta efectuada, se relaciona con la necesidad de profundizar en la implementación y evaluación de programas 
formativos, con los énfasis que se han mencionado en este documento. Es imprescindible conocer los facilitadores y obstaculizadores de experiencias como éstas, que sirvan de insumo para los cambios que se necesitan en este ámbito.

\section{Referencias bibliográficas}

Ávalos, B. (2003). "Las instituciones formadoras de docentes y las claves para formar buenos docentes". Ponencia presentada en "II Encuentro Internacional De La Red De Formación Docente De América Latina y El Caribe, El Desafio De Formar Los Mejores Maestr@s,", San Pedro Sula, Honduras.

Ávalos, B. (2006). Formación inicial docente: Nudos críticos. Recuperado el 5 de enero del 2008 desde www.mideuc.cl/docs/Formacion_Docente_ Inicial.pdf

Carranza, G. \& Reyes, M. (2005). "El saber pedagógico desde la visión de las instituciones formadoras de profesores". Sistematización $2^{\circ}$ encuentro nacional sobre formación docente.

Contreras, I.; Rittershaussen, S.; Montecinos, C.; Solís M.; Walker H. \& Núñez C. (2008, junio). "Caracterización de la formación práctica en las carreras de pedagogía en educación media: estudio de casos múltiples". Ponencia presentada en $I^{\circ}$ Congreso Internacional sobre Profesorado Principiante e Inserción Profesional a la Docencia. Sevilla, España.

Cornejo, J. (2003). "El pensamiento reflexivo entre profesores". Revista Pensamiento educativo. Pontificia Universidad Católica de Chile. 32, p. 58-79

De Tezanos, A. (2005). "El camino de la profesionalización docente". Revista Prelac, 1, p. 60-77.

Díaz, V. (2005). "Teoría emergente en la construcción del saber pedagógico". Revista Iberoamericana de Educación, 37, p. I-19.

Eyzaguirre, B. \& Le Foulon, C. (200I). "La calidad de la educación chilena en cifras". Estudios Públicos, 84, p. 85-225.

Gauthier, C. (2006). "La política sobre formación inicial de docentes en Québec". Revista de Educación, 340, p. 165-185.

Harford, J. \& MacRuairc, G. (2008). "Engaging student teachers in meaningful reflective practice". Teaching and teachers education, 24, p. 1884-1892.

Ibáñez, N. (2007). "La voz de los expertos”. En M. Nervi, \& H. Nervi (Eds.), ¿Existe la pedagogía? Hacia la construcción del saber Pedagógico Santiago, Universitaria: (p. 65-99). 
Cambios al currículum de la formación inicial de profesores: Resignificando el saber pedagógico

Jay \& Johnson (2002). "Capturing complexity: a typology of reflective practice for teacher education". Teaching and Teacher Education, I8, p. 73-85.

Marcelo, C. (2002). "Los profesores como trabajadores del conocimiento. Certidumbres y desafíos para una formación a lo largo de la vida”, Educar, 30, p. 27-56.

Ministerio de Educación (200I). Estándares de desempeño para la formación inicial docente. Santiago, Chile.

Ministerio de Educación (2005). Informe Comisión formación inicial docente. Santiago, Chile.

Ministerio de Educación (2007). Informe Nacional SIMCE. Santiago, Chile.

Moreno, N.; Rodríguez, A.; Torres, J.; Mendoza, N. \& Vélez, L. (2006). Tras la buella del saber pedagógico. Universidad Nacional Pedagógica.

Murillo, P. (2002). "Educación, sociedad y conocimiento: el peso de la realidad en la formación”. Perspectivacep. Revista de los Centros de Profesorado de Andalucía, 4, p. 93-109.

Nervi, M. \& Nervi. H. (2007). ¿Existe La Pedagogía? Hacia la Construcción del Saber Pedagógico. Santiago: Universitaria.

OECD (2003). Informe de la Educación Chilena. OECD/Ministerio de Educación, Santiago.

Ottone, E. \& Hopenhayn, M. (2007). "Los desafíos ante la sociedad del conocimiento”. Revista Pensamiento Educativo, 40, p. I3-29.

Salazar, S. (2005). "El conocimiento pedagógico del contenido como categoría de estudio de la formación docente". Actualidades investigativas en educación, 5, p. I-I6.

Shulman, L. (1987; 200I). “Conocimiento y Enseñanza”. Estudios Públicos, 83, p. 163-196.

Torres, M. (1996). "Formación Docente: Clave de la Reforma Educativa". En UNESCO - OREALC. Nuevas formas de aprender y enseñar (p. 5-35). Santiago, Chile: UNESCO.

Valenzuela, I. (noviembre, 2006). "Hacia la construcción del saber especializado del profesor: el saber pedagógico”. Ponencia presentada en Seminario Modelos de formación docente: El problema del saber pedagógico y el saber disciplinario, Chillán, Chile. 